\title{
Graphene-Fiber Biochemical Sensors: Principles, Implementations, and Advances
}

\author{
Ning $\mathrm{AN}^{1 \dagger}$, Chenye QIN ${ }^{1,2 \dagger}$, Yiwei LI ${ }^{1}$, Teng TAN ${ }^{1,2}$, Zhongye YUAN ${ }^{1}$, \\ Hao ZHANG ${ }^{1}$, Yu WU ${ }^{1 *}$, Baicheng $\mathrm{YAO}^{1 *}$, and Yunjiang $\mathrm{RAO}^{1,2^{*}}$ \\ ${ }^{1}$ Key Lab of Optical Fiber Sensing \& Communications, University of Electronic Science \&Technology of China, \\ Chengdu 611731, China \\ ${ }^{2}$ Research Center for Optical Fiber Sensing, Zhejiang Laboratory, Hangzhou 310000, China \\ ${ }^{\dagger}$ These authors contribute equally to this work \\ *Corresponding authors: Baicheng YAO, Yu WU, and \\ E-mail: yaobaicheng@uestc.edu.cn, \\ Yunjiang RAO \\ wuyu@uestc.edu.cn, and yjrao@uestc.edu.cn
}

\begin{abstract}
Single atomically thick graphene, with unique structural flexibility, surface sensitivity, and effective light-mater interaction, has shown exceptional advances in optoelectronics. It opens a door for diverse functionalized photonic devices, ranging from passive polarizers to active lasers and parametric oscillators. Among them, graphene-fiber biochemical sensors combine the merits of both graphene and fiber structures, demonstrating impressively high performances, such as single-molecule detectability and fast responsibility. These graphene-fiber biochemical sensors can offer tools in various applications, such as gas tracing, chemical analysis, and medical testing. In this paper, we review the emerging graphene-fiber biochemical sensors comprehensively, including the sensing principles, device fabrications, systematic implementations, and advanced applications. Finally, we summarize the state-of-the-art graphene-fiber biochemical sensors and put forward our outlooks on the development in the future.
\end{abstract}

Keywords: Graphene; fiber sensors; biochemical sensing

Citation: Ning AN, Chenye QIN, Yiwei LI, Teng TAN, Zhongye YUAN, Hao ZHANG, et al., "Graphene-Fiber Biochemical Sensors: Principles, Implementations, and Advances," Photonic Sensors, 2021, 11(1): 123-139.

\section{Introduction}

Fiber sensors, developed since the 1970s, with remarkable compatibility, unique sensitivity, and insurmountable tolerance for hostile environment, have been revolutionizing our life. Fiber sensors are applicable in the real world with high sensitivity by associating external information with optical parameters, including light intensity [1], wavelength [2], and phase [3]. In recent years, different combinations between fiber structures and two-dimensional materials, further broaden the capability of fiber sensors into biochemical applications [4-9]. Graphene, as a pioneer member of two-dimensional materials, has shown remarkable advances ranging from materials science [10], physics, chemistry [11, 12], and electronics [13] to mechanics [14], due to its linear band structure and atomic thickness [15], as shown in Fig. 1(a). In the branch of photonics, graphene has been widely

Received: 3 November 2020 / Revised: 16 December 2020

(C) The Author(s) 2021. This article is published with open access at Springerlink.com

DOI: $10.1007 / \mathrm{s} 13320-021-0617-6$

Article type: Review 
applied in various optoelectronic devices, building upon the excellent electrical conductivity, ultra-high carrier mobility, extremely strong nonlinearity, and unique carrier density tunability [16-19]. Among the graphene based photonic devices, the biochemical sensor is one of the most rapidly developed

(a)

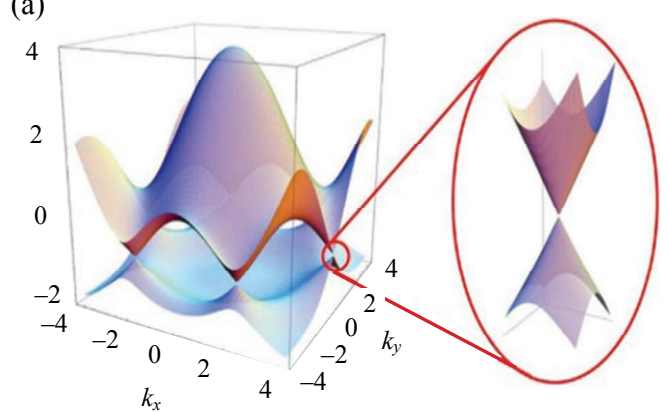

(c)

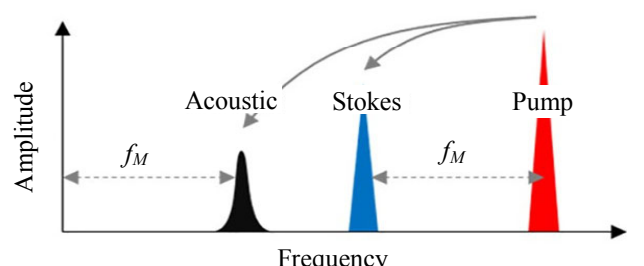

Frequency
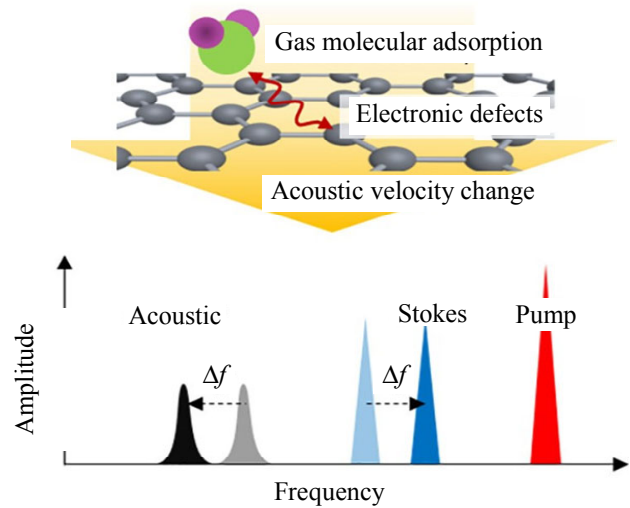

applications. Via continuously optimizing the graphene materials, the fiber structures, and their combinations, the performance of graphene-fiber biochemical sensors is pushed to new height constantly, such as ultrahigh sensitivity $[20,21]$ and selectivity [5].

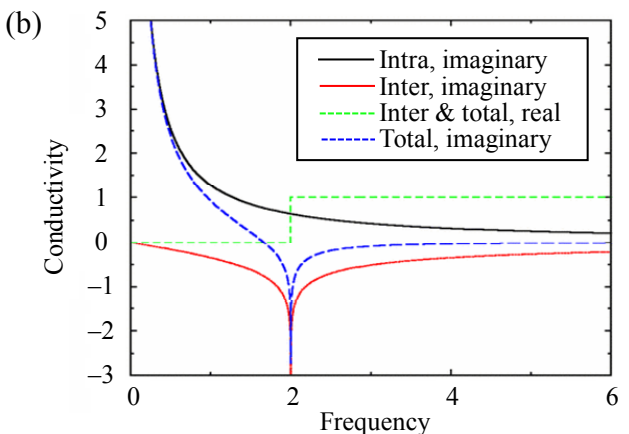

(d)
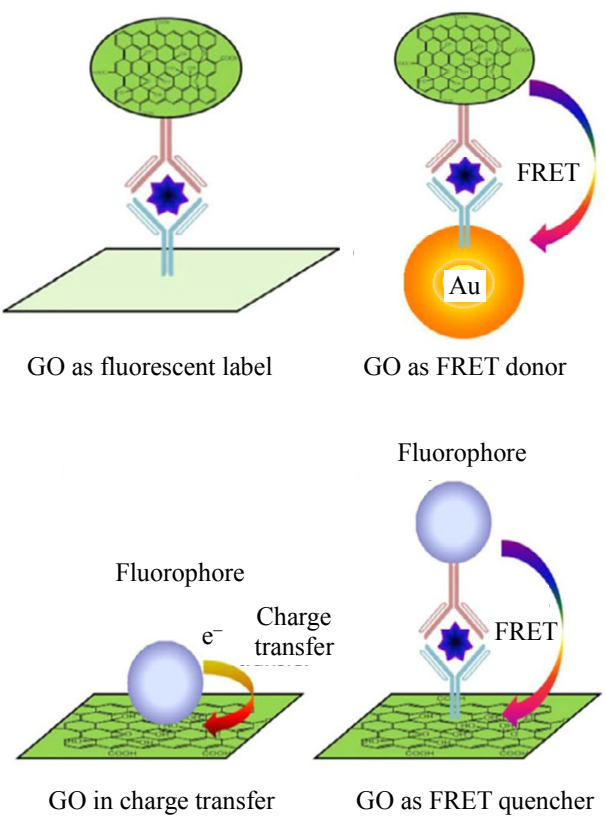

Fig. 1 Graphene sensing mechanism: (a) graphene single-molecule structure and energy band linear distribution [22], (b) dynamic conductivity of graphene for intraband and interband [23], (c) "electron-phonon-photon" process in graphene enhanced Brillouin optomechanical micro resonator [20], and (d) schematic illustration of FRET sensing process based on GO [24].

In this review, firstly, we elaborate the sensing principles of graphene-fiber sensors and analyze the advantages and disadvantages of these technical routes. Secondly, we illustrate the preparation of graphene materials and show how to integrate them onto fiber platforms. Thirdly, we review the state-of-the-art graphene-fiber biochemical sensors, which work in gas or liquid environment. Finally, we present our outlooks about the future perspectives.

\section{Sensing principles of graphene-fiber hybrids}

In general, fiber sensors based on graphene typically rely on their linearly gapless Dirac-Fermi distribution [25]: 


$$
E_{F}= \pm \hbar v_{F}|\mathbf{k}|
$$

where $v_{F}$ is the Fermi velocity of graphene, $\mathbf{k}$ is the wave vector, and $\hbar$ is the reduced Planck constant. Graphene demonstrates excellent electronic conductivity, $v_{F}$ is $1 / 300$ of the light, and the carrier mobility can reach up to $2 \times 10^{6} \mathrm{~cm}^{2} \cdot \mathrm{V}^{-1} \cdot \mathrm{s}^{-1}$, which corresponds to resistance of $10^{-6} \Omega$. Besides, its Fermi level can also be quickly and efficiently modulated when the external environment parameters change. In optics, such environmentally dependent Fermi level leads to optical responses of graphene. Based on the different responses, the principles of graphene-fiber sensors can mainly be divided into linear response and nonlinear response.

\subsection{Sensing based on linear response}

Linear response of the composite waveguide is the most traditional sensing mechanism of graphene-fiber sensing. Typically, the linear response of graphene-fiber sensors generally relies on the change in refractive index (RI). The complex refractive index expressions of the graphene-fiber sensors are as follows [26]:

$$
\begin{gathered}
n_{g, r}=\left[\begin{array}{c}
2 \varepsilon_{g, r}\left(\frac{\varepsilon_{g, r}}{2}+\frac{\sqrt{\varepsilon_{g, r}{ }^{2}-\varepsilon_{g, i}^{2}}}{2}\right)^{1 / 2}- \\
2\left(\frac{\varepsilon_{g, r}}{2}+\frac{\sqrt{\varepsilon_{g, r}{ }^{2}-\varepsilon_{g, i}^{2}}}{2}\right)^{3 / 2}
\end{array}\right] / \varepsilon_{g, i} \\
n_{g, i}=\left[\frac{\sqrt{\varepsilon_{g, r}{ }^{2}+\varepsilon_{g, i}^{2}}}{2}-\frac{\varepsilon_{g, r}}{2}\right]^{1 / 2}
\end{gathered}
$$

where $n_{g, r}$ and $n_{g, i}$ are the real and imaginary parts of complex refractive index, and $\varepsilon_{g, r}$ and $\varepsilon_{g, i}$ are the real and imaginary parts of effective optical permittivity, respectively. In an ultra-thin graphene film, the permittivity can be expressed as

$$
\varepsilon_{g}=\frac{-\sigma_{g, i}+\mathrm{i} \sigma_{g, r}}{2 \pi f \Delta}
$$

where $\sigma_{g, r}$ and $\sigma_{g, i}$ are the real and imaginary parts of conductivity, $\Delta$ is the graphene monolayer thickness, and $f$ is the optical frequency. As above all, the complex refractive index of graphene is determined by its conductivity. Approximated by the Drude model, the conductivity of graphene could be divided into interband conductivity and intraband conductivity to describe different types of photoelectron interactions, and the corresponding conductivity curve is shown in Fig. 1(b):

$$
\begin{gathered}
\sigma_{g, \text { intra }}=\frac{\mathrm{ie}^{2} E_{F}}{\pi \hbar\left(2 \pi f+\frac{\mathrm{i}}{\tau}\right)} \\
\sigma_{g, \text { inter }}=\frac{\mathrm{ie}^{2} E_{F}}{4 \pi \hbar} \ln \left[\frac{2\left|E_{F}\right|-\hbar\left(2 \pi f+\frac{\mathrm{i}}{\tau}\right)}{2\left|E_{F}\right|+\hbar\left(2 \pi f+\frac{\mathrm{i}}{\tau}\right)}\right]
\end{gathered}
$$

where $e=-1.6 \times 10^{-19} \mathrm{C}, \hbar=1.05 \times 10^{-34} \mathrm{~J} \cdot \mathrm{s}, f$ is the optical frequency, $\tau \approx 10^{-13} \mathrm{~s}$ is the carrier relaxation lifetime, $\hbar$ is Planck's constant, and $E_{F}$ is the quasi Fermi level, directly determined by the external bias. The Fermi level in graphene changes as

$$
E_{F}(n)=\hbar\left|\mathbf{v}_{F}\right| \sqrt{\pi n}
$$

where $n$ is the carrier concentration [27]. When molecules are absorbed on the surface of graphene, the adsorbed molecules will offer or take carriers and then change the carrier concentration, which makes the Fermi level tuned. Finally, the modulation of the refractive index is transformed into the modulation of light, such as intensity [28] and phase [29], which can be used to obtain sensing information by demodulating the output signal. Based on this principle, many sensing applications can work in both gas and liquid environments, with simple setups.

Furthermore, to enhance light-graphene interaction in graphene-fiber structures, surface plasmons are introduced due to their strong confinement for light [30]. The new technical method is sensitive to the real part of refractive index of the surface material, so a small change in refractive index will lead to the shift of the resonance peak [31]. The sensors based on graphene plasmon show higher sensitivity and great 
significance for biochemical detection in the mid-infrared [32], but due to the large loss of plasmon [33], it is currently difficult to be fully applied to other practical devices.

In general, refractive index susceptibility is one of the most important mechanisms of graphene-fiber in biochemical detection, but the sensitivity and resolution of this scheme is limited by the spectral resolution of the optical interference and the linear loss of the passive devices.

\subsection{Sensing based on nonlinear response}

Different from linear response, graphene-fiber sensors based on nonlinear response show good sensing performance due to their specificity. Graphene has rich nonlinear phenomena including the saturable absorption, second-order nonlinearity, and third-order nonlinearity [13]. The contact between the external molecule and graphene will cause changes in nonlinear parameters of graphene, thereby affecting the intensity or efficiency of graphene nonlinear signals such as four-wave-mixing (FWM) and stimulated Brillouin scattering (SBS) [20, 21]. For instance, Fig. 1(c) shows an example of the graphene enhanced Brillouin scattering in a microresonator to achieve ammonia sensing, expanding the "electron-photon" interaction to "electron-phonon-photon". In this work, optomechanical resonance is generated via Brillouin phase matching and nonlinear gain:

$$
\begin{aligned}
\frac{f_{M}}{v_{A}} & =\frac{2 \pi f_{p} n_{p}}{c}-\frac{2 \pi f_{s} n_{s}}{c} \\
g_{B} & \propto \frac{4 \pi^{2} \gamma_{e}^{2}}{n_{p} c \lambda_{p}^{2} \rho_{0} v_{A} \Gamma_{B}}
\end{aligned}
$$

where $v_{A}$ is the acoustic velocity, $c$ is the light velocity in vacuum, $n_{p}$ and $n_{s}$ are the effective indexes of the pump mode and the generated Stokes mode, $f_{p}$ and $f_{s}$ are the pump frequency and the Stokes frequency, $f_{M}$ is the acoustic mechanical resonance frequency, $\gamma_{e}$ is the electro-strictive coefficient, $\rho_{0}$ is the density of the material, and $\Gamma_{B}$ is the lifespan of the phonons.
Gas detection relies on the phase matching of the optomechanical modes. Spectral shift of the $f_{s}$ is determined by $v_{A}$ majorly:

$$
\frac{\partial f_{s}}{\partial x}=\frac{2 \pi n_{s}-2 \pi n_{p}}{\left(c-2 \pi v_{A} n_{s}\right)^{2}} \frac{\partial v_{A}}{\partial x} f_{p}
$$

where $x$ is the gas concentration. This equation reveals that when $v_{A}$ decreases with the gas concentration, $f_{s}$ increases accordingly, which also verifies that $f_{M}=f_{p}-f_{s}$ decreases when launching a higher gas concentration. And a smaller $n_{s}$ brings the higher sensitivity when $n_{p}$ and $f_{p}$ are fixed, which means a higher-order mode is helpful for the sensitivity enhancement [20].

Different from the nonlinear sensing mechanism mentioned above, recently, a high-sensitivity gas sensing mechanism based on the relationship between the FWM efficiency and Fermi level was proposed. There is a steep change (increase/decrease) of the FWM efficiency when the Fermi level approaches $0.4 \mathrm{eV}$. Exploiting this feature, there is a relationship between the FWM efficiency and the adsorption of gas molecules, because the polar gas molecules could change the Fermi level of graphene. When graphene is predoped around $0.4 \mathrm{eV}$, the sensor achieves the highest sensitivity [21].

Compared with the sensing scheme based on linear response, such a sensing scheme based on nonlinearities can avoid linear noises in devices, thus enables optical sensors to demonstrate high purity in the signal and show a potential for high-precision and high-resolution quality sensors.

\subsection{Fluorescence resonance energy transfer}

Different from the principles mentioned above, fluorescence resonance energy transfer (FRET) is a distance-dependent physical process by whose energy is transferred non-radiatively from an excited molecular fluorophore (the donor) to another fluorophore (the acceptor) [34]. It relies on the fluorescence spectra and distance between the donor and acceptor rather than the Fermi level modulation. 
Figure 1(d) shows the FRET process based on graphene oxide (GO) [24]. Compared with the conventional biosensors based on FRET, graphenefiber sensors do not need to be labeled and are simple and safe to prepare. Moreover, graphene and its derivatives have a large specific surface area and could be used as a quencher for a variety of fluorescent groups to achieve multi-channel detection. When serving as a general quencher, graphene and its derivatives can simultaneously quench the fluorescence of multiple fluorophores with high efficiency, low background noise, and high signal to noise ratio. In some cases, graphene and its derivatives can also generate fluorescence signals. No matter what role the graphene plays in the process of contacting and interaction with other molecules, the energy exchange between the fluorescent donor and the acceptor will occur and the fluorescence intensity will be affected by the concentration of the sensing substance. By detecting the fluorescent intensity, graphene-fiber sensors based on FRET could be realized. This scheme enables graphene-fiber sensors to have remarkable chemical selectivity. But compared with phase demodulation based on RI, the intensity measurement shows lower resolution. Moreover, the FRET based sensors work in liquid typically, they are more widely used for bio detections than gas tracing. Hence, combining the FRET and interferometer in one sensor device could be an optimized method [5, 35].

\section{Fabrication of graphene-fiber hybrids}

\subsection{Synthesis of graphene-based nanomaterials}

In biochemical sensing applications, people use types of graphene derivations, such as graphene, GO, reduced graphene oxide (rGO), and partially reduced graphene oxide (prGO) [24]. Related to pristine graphene, GO has a similar hexagonal carbon structure but contains rich functional groups (such as hydroxyl and carboxyl). The structural diagrams are shown in Fig.2(a), and from left to right are pristine graphene, GO, and rGO [36].

The synthesis methods of these materials are different due to disparity in structures and properties. At present, chemical vapor deposition method (CVD) is a widely used method to prepare large-area and high-quality graphene with controllable layer number [37]. Figure 2(b) illustrates the schematic process of growing graphene on a $\mathrm{Cu}$ substrate via CVD. Carbon sources such as methane or ethanol are used to decompose in a dilute $\mathrm{H}_{2}$ environment at $1000^{\circ} \mathrm{C}$, and the carbon atoms are released on the $\mathrm{Cu}$ surface and diffused into the metal, eventually, precipitating and forming a continuous graphene film on the surface of the $\mathrm{Cu}$ layer when the substrate is cooled down. Graphene produced by the CVD has fewer defects and excellent uniformity in the inch-size scale, as shown in Fig. 2(c) [26]. Besides, graphene with high quality can also be obtained by non-growth methods, like mechanical exfoliation and liquid phase exfoliation [38, 39], but the size and the number of layers are difficult to control.

The rich functional groups on the surface of GO could bind to a variety of biochemical molecules, enhancing the sensitivity of graphene-fiber sensors. In most cases, GO is obtained by Hummer's method [40], which introduces the oxygen-containing functional group to graphite through oxidizing agent, such as the mixture of concentrated sulfuric acid, sodium nitrate, and potassium permanganate. After surface functionalization, GO could be combined with fiber to obtain fiber sensors with specific recognition. Furthermore, by reducing GO, rGO could be obtained, which still has a few oxygen groups but achieves similar properties to graphene film (such as non-dispersive). This enables it to keep stable as a tight film on fiber in the aqueous environment, meanwhile demonstrating biochemical bonding capability [35]. The whole flow is shown in Fig. 2(d) [40]. 
(a)

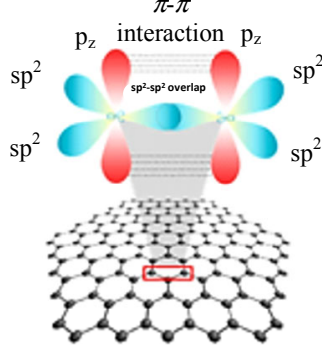

Graphene

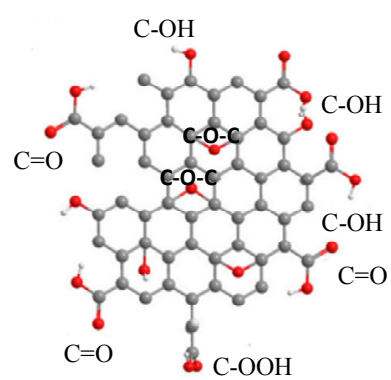

Graphene oxide

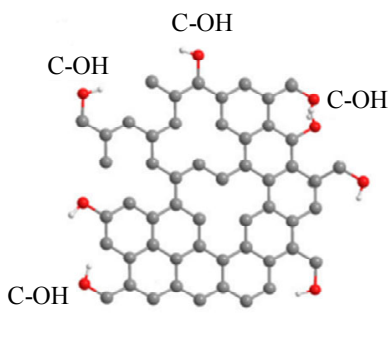

Reduced

graphene oxide (b)

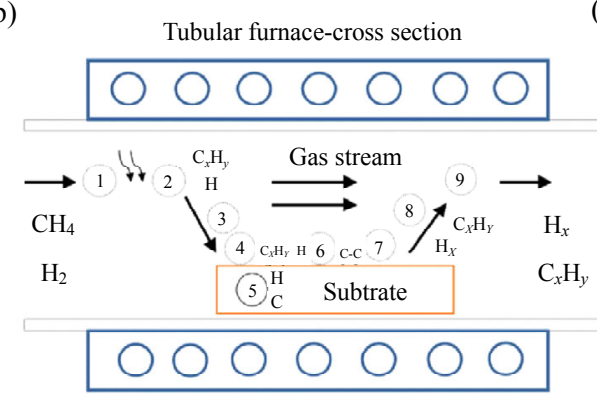

(c)

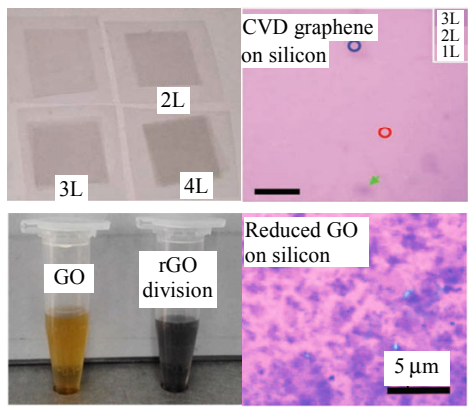

(e)

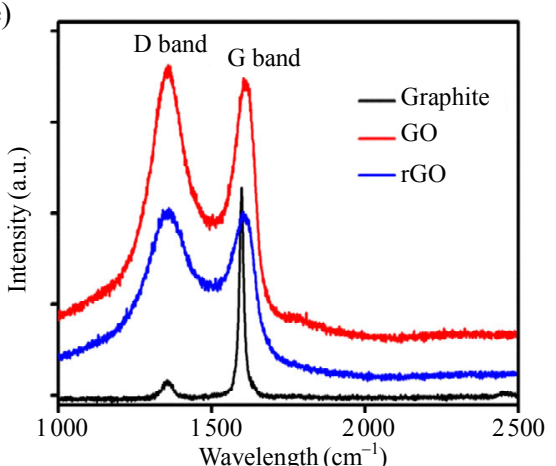

Fig. 2 Synthesis of graphene and its derivatives: (a) structures of graphene-based materials [pristine graphene(left), GO(middle), and rGO(right)] [36], (b) illustration of the CVD method for growing graphene on the Cu substrate [41], (c) picture of graphene grown from CVD, and GO, rGO division, the deposition film on silicon substrate are also shown [26], (d) flow chart of GO and rGO preparation [40], and (e) measured Raman spectra of graphite, GO, and rGO [42].

Besides, by decreasing the reduction time, prGO could be obtained. Compared with rGO, prGO contains more oxygen groups. So, it could be used to improve the sensitivity and selectivity for biochemical sensors [5, 35]. The Raman spectra of graphite, GO, and rGO are shown in Fig.2(e). The intensity ratio between the disorder induced $\mathrm{D}$ band and the Raman allowed $\mathrm{G}$ band $\left(I_{D} / I_{G}\right)$ could reveal the difference among graphite, GO, and rGO. Compared with graphite, an increase in $I_{D} / I_{G}$ of GO demonstrates the grafting of oxygen containing functional groups to the graphitic planes. After reduction, a decrease in $I_{D} / I_{G}$ shows that most of the oxygen-containing functional groups have been removed. But the intensity ratio of $\mathrm{rGO}$ is higher than that of graphite, because the $\mathrm{sp}^{2}$ domains are smaller than those of graphite [42].

\subsection{Installation of graphene-fiber hybrids}

Generally, graphene-fiber hybrids are mainly prepared by transferring or depositing graphene on the specific fiber structures, such as microfiber, 
D-shaped fiber, photonic crystal fiber (PCF), and microcavity. For the graphene transfer method, wet transfer is the most mature method for the fabrication of graphene-fiber hybrids, which is mainly used for the microfiber, D-shaped fiber, and fiber endface, as shown in Fig. 3(a) [43, 45]. The specific process of wet transfer is as follows: Firstly, the polymethyl methacrylate (PMMA) is spin-coated on the upper surface of the graphene/copper composite layer prepared by the CVD method, and then the PMMA/graphene/copper composite layer is placed in the $\mathrm{FeCl}_{3}$ solution to dissolve the copper layer. The PMMA/graphene composite film with the copper layer removed will float on the surface of the solution. Secondly, the PMMA/graphene flexible film is soaked and cleaned with deionized water, and the PMMA/graphene composite film could be combined with the waveguide. Finally, the fiber with the PMMA/graphene film is placed in acetone or acetone vapor to remove the PMMA to obtain a graphene/fiber sensor [26].

Besides, the depositing method is mainly used in the preparation of graphene derivative fiber sensors, such as GO and rGO. For example, Fig. 3(b) shows the process of depositing rGO on the surface of the microfiber. The process requires oxidizing graphene powder to GO with strong oxidizer (such as potassium permanganate) and immersing the microfiber in the GO dispersion. rGO could be obtained by reducing GO with reductant such as vitamin C. The rGO film deposited on the surface of microfiber could be optimized by controlling the reduction time and temperature [26].

In addition to the two main methods mentioned above, there are other optional methods for specific fiber structure. For example, Chen et al. [44, 46, 47] proposed a method of directly growing graphene via CVD with high crystallinity on the surface of PCF, as shown in Fig.3(c).

(a)
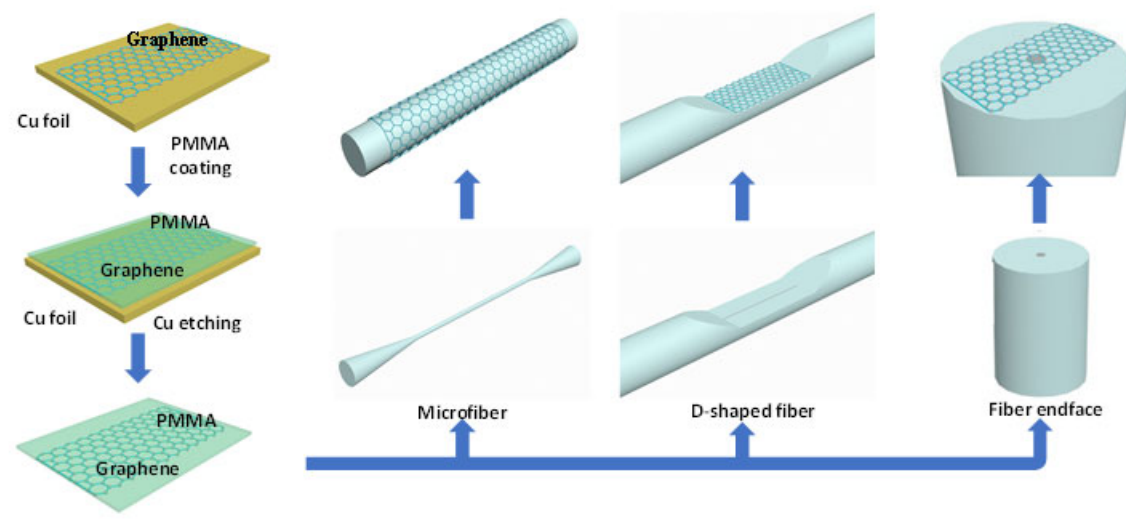

(b)
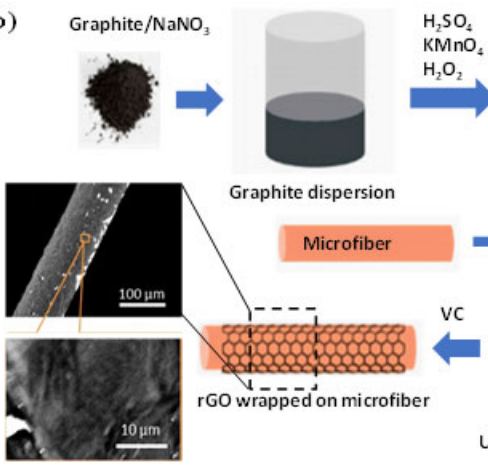

(1)

(c)

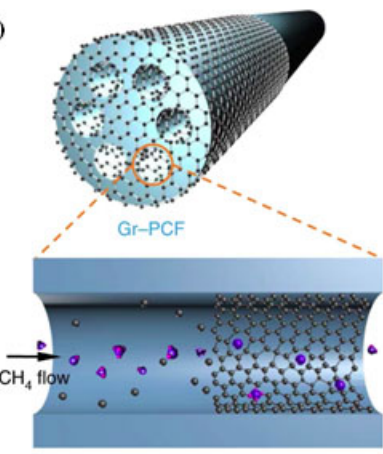

UItrasonic GO solution

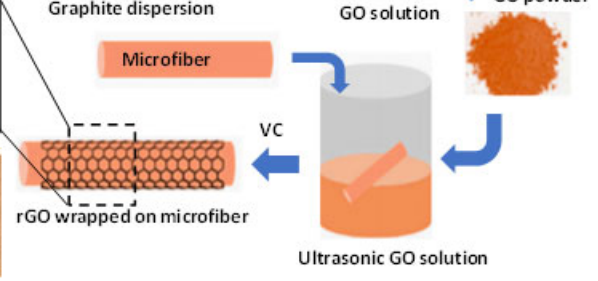

Fig. 3 Fabrication of graphene-based fiber structures: (a) schematic diagram of wet transfer combining graphene with the microfiber, D-shaped fiber, and fiber endface [43], (b) flow of depositing method based on GO reduction [20], and (c) schematics of graphene-PCF grown by the CVD method [44]. 


\section{Graphene-fiber biochemical sensing implementations and applications}

In this section, we systematically classify the sensing objects of biochemical sensors into gas and liquid categories. Among them, gases include gas molecules, water molecules, and volatile substances, while liquids mainly include carbohydrates, biomacromolecules, aqueous ions, and other clinically relevant analytes [48-52]. In the following content, we will introduce the latest research progress of graphene-fiber sensors, including different sensing structures and sensing targets, and analyze their sensing performances.

\subsection{Gas sensors}

Gas sensor based on graphene was first reported in 2012, and a hybrid graphene-microfiber waveguide structure for acetone gas detection was proposed [53]. The adsorption of gas molecules would dramatically dampen the optical transmission, resulting in a maximum sensitivity of about $0.3 \mathrm{~dB} / \mathrm{ppk}$ and a regeneration time of several minutes. For the better sensing performance, the same group developed an ammonia sensor by using a Mach-Zehnder interferometer (MZI) in 2014, in which the graphene-microfiber was used to form one of arms. The interference wavelength shift of the MZI was related to the concentration of $\mathrm{NH}_{3}$ gas, showing a resolution of $0.3 \mathrm{ppm}$ and a response time of $0.5 \mathrm{~s}$ [54]. And in the same year, they further reported graphene Bragg gratings on the microfiber for gas detection [55]. Different from the research mentioned above, graphene was wrapped rather than attached on the microfiber, which greatly increased the contact area between the graphene and microfiber. Its spectral sensitivity for $\mathrm{NH}_{3}$ gas detection was $2 \mathrm{pm} / \mathrm{ppm}$ in the gas concentration range from $0 \mathrm{ppm}$ to $10 \mathrm{ppm}$ with the maximum sensitivity of $\sim 0.5 \mathrm{ppm}$. Besides, they demonstrated a graphene based microfiber multimode interferometer for gas sensing [56], achieving a sensitivity of $\sim 0.1 \mathrm{ppm}$ for $\mathrm{NH}_{3}$ gas detection and $\sim 0.2 \mathrm{ppm}$ for $\mathrm{H}_{2} \mathrm{O}$ vapor detection with the good repeatability, as shown in Fig. 4(a). Furthermore, taking advantage of both the graphene-induced evanescent field enhancement and the in-fiber multimode interferometer, they proposed a graphene-coated D-shaped fiber (GDF) chemical gas sensor to promote the maximum sensitivities for $\mathrm{NH}_{3}$ and $\mathrm{H}_{2} \mathrm{O}$ gas detection to $\sim 0.04 \mathrm{ppm}$ and $\sim 0.1 \mathrm{ppm}$ with a response time of $\sim 20$ s [57].

Based on the same method of interferometric wavelength drift monitoring, some novel structures have been proposed. For instance, Feng et al. [58] reported a $\mathrm{H}_{2} \mathrm{~S}$ sensor based on the graphene-coated tapered PCF-MZI, which was capable of detecting hydrogen sulfide gas from $0 \mathrm{ppm}$ to $45 \mathrm{ppm}$ with a response time of $60 \mathrm{~s}$ and showed a sensitivity of $0.03143 \mathrm{~nm} / \mathrm{ppm}$, as illustrated in Fig. 4(b). And Pawar et al. [59] demonstrated a $\mathrm{Fe}_{3} \mathrm{O}_{4}$-graphene nanocomposite coated Fabry-Perot interferometer (FPI) for ammonia gas detection, achieving a sensitivity of $36 \mathrm{pm} / \mathrm{ppm}$ with gas detection limit of $7 \mathrm{ppb}$. In addition, the utilization [60] and doping modification $[59,61,62]$ of graphene derivatives, as two important research directions, have been widely used to improve the sensitivity and selectivity of graphene-fiber sensors. By adopting the similar structure but different material in [55], Sridevi et al. designed a $\mathrm{NO}_{2}$ sensor using $\mathrm{rGO}$ coated clad etched fiber Bragg grating. In this work, the change in the refractive index of $\mathrm{rGO}$ by charge transferring between adsorbed $\mathrm{NO}_{2}$ and $\mathrm{rGO}$ caused the shift in Bragg wavelength. The result showed a lower detection limit of $0.5 \mathrm{ppm}$ with $0.8 \mathrm{pm} / \mathrm{min}$ sensitivity, as shown in Fig. 4(c) [60]. It also mentioned that sensor accuracy could be improved to sub ppb level and the response time could be decreased by having multiple gratings in the same fiber.

Thanks to the tight adsorption capacity of $\mathrm{Pt}$ particle surface to $\mathrm{NH}_{3}$ gas molecules, Yu et al. [62] doped GO with $\mathrm{Pt}$ to improve $\mathrm{NH}_{3}$ absorption. The result showed a sensitivity of $10.2 \mathrm{pm} / \mathrm{ppm}$ with fast 
response time and good reversibility from $0 \mathrm{ppm}$ to $60 \mathrm{ppm}$, which was three times higher than the sensitivity without Pt-decorated nanoparticles, as shown in Fig. 4(d). Because of the moisture sensitivity of the Poly vinyl alcohol (PVA), Wang et al. [63] demonstrated a humidity sensor based on an in-fiber MZI coated with the GO/PVA composite film for further improving the sensing performance. It showed an maximum sensitivity of $0.193 \mathrm{~dB} / \%$ $\mathrm{RH}$ and a good stability under the relative humidity range of $25 \%-80 \%$. Similar to the method mentioned above, Fu et al. [64] also introduced zinc oxide nanoparticle incorporated $\mathrm{GO}(\mathrm{GO}-\mathrm{ZnO})$ to serve as the sensing material in the multimode microfiber interferometer, and the surface evanescent field of the microfiber could be significantly enhanced by the GO-ZnO coating. This sensor exhibited the high sensitivity for $\mathrm{NH}_{3}$ ranging from $4 \mathrm{ppm}$ to $140 \mathrm{ppm}$ and demonstrated a good repeatability and a recovery of less than $90 \mathrm{~s}$. Besides, graphene-fiber sensors could also be used to detect volatile organic compounds. For example, Zhang et al. [65] proposed a GO coated microfiber ethanol vapor sensor. It exhibited a sensitivity of $0.138 \mathrm{~nm} / \mathrm{ppm}$ in the concentration range from $0 \mathrm{ppm}$ to $80 \mathrm{ppm}$.

(a)
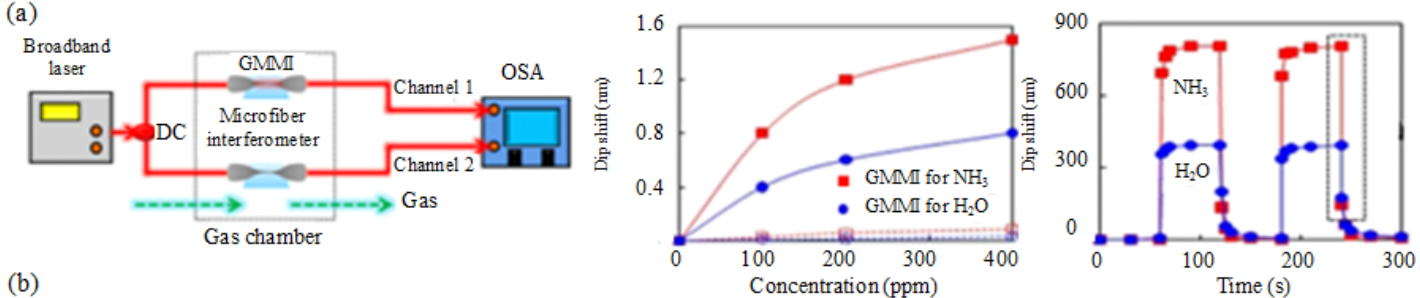

(b)
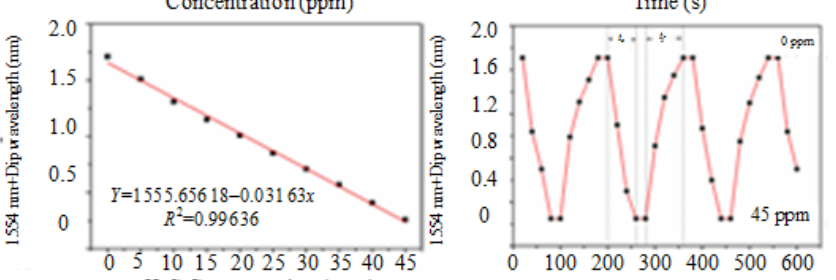

(c)
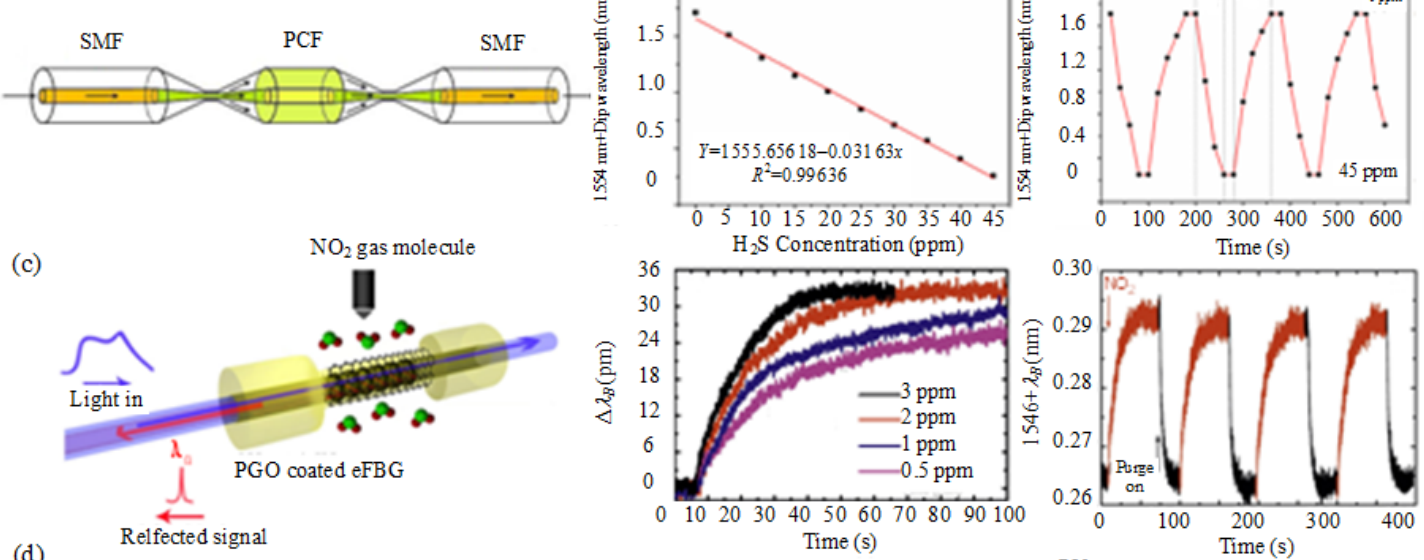

(d)
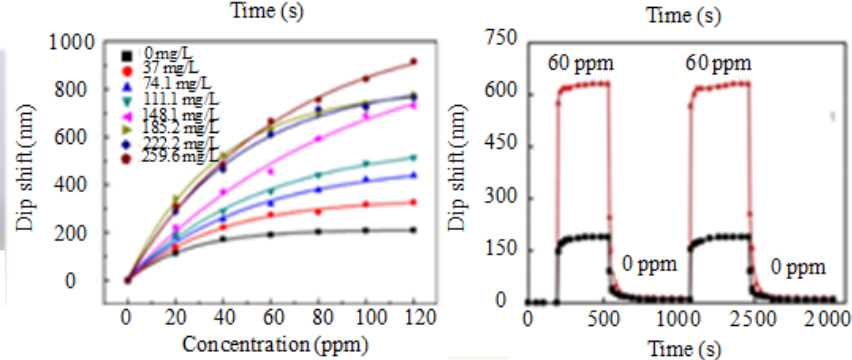

Fig. 4 Graphene-fiber gas sensors: (a) graphene/microfiber sensor for $\mathrm{NH}_{3}$ and $\mathrm{H}_{2} \mathrm{O}$ [56], (b) graphene/PCF sensor for $\mathrm{H}_{2} \mathrm{~S}$ [57], (c) rGO/etched FBG sensor for $\mathrm{NO}_{2}$ [60], and (d) Pt nanoparticle-incorporated $\mathrm{GO} /$ microfiber sensor for $\mathrm{NH}_{3}$ [62] . 
In addition to optimizing fiber structure and materials to improve the performance, some new sensing mechanisms are introduced in recent years [20, 21]. Typically, Yao et al. [20] proposed a microfiber interrogated whispering gallery mode (WGM) optomechanical gas sensor, which expanded "electron-photon" interaction in conventional graphene based optical sensors to "electron-phonon-photon" interaction. It achieved an unprecedented high sensitivity (1 ppb) for $\mathrm{NH}_{3}$ gas detection and a wide dynamic range over five orders of magnitude. More recently, An et al. [21] demonstrated a scheme to generate ultrasensitive down-conversion four-wave-mixing (FWM) in a graphene bipolar-junction-transistor heterogeneous D-shaped fiber for gas sensing and found that when the graphene $E_{F}$ was predoped at $\sim 0.4 \mathrm{eV}, \mathrm{FWM}$ in the device became extremely sensitive to molecular adsorption/ desorption. And they achieved real-time individual gas molecule detection in vacuum.

\subsection{Biosensors}

For the detection of substances in a liquid environment, graphene and its derivatives can still maintain excellent sensing properties because of their large surface area, high electrical conductivity, and unique stability in aqueous media. In addition, graphene can be also used as a molecular scaffold to fix target biomolecular analytes on the required functional groups, resulting in selectivity of biosensors. According to different applications, liquid biochemical sensors based on graphene-fiber composite waveguides could be mainly divided into the following types: glucose [31], protein [66], deoxyribonucleic acid (DNA) [67], and other small molecules [68].

For diabetic patients, the continuous and accurate detection of glucose concentration in blood is clinically important, and many researchers are currently working on high-quality glucose concentration sensors. Since the concentration of glucose is linearly related to the refractive index of the glucose solution, some researchers proposed to use the refractive index sensitive properties of the graphene-fiber composite waveguide to prepare a glucose sensor. For example, Zhang et al. [69] proposed a highly sensitive fiber surface plasmon resonance (SPR) sensor based on a D-shaped fiber modified by graphene for implantable continuous glucose monitoring. In this work, they engraved the long-period grating (LPG) onto the fiber core to incorporate a temperature sensor to eliminate the influence on the refractive index of glucose, which improved the accuracy of detection via compensating for the temperature drift of the SPR spectrogram, as shown in Fig. 5(a). The result showed that the sensor with monolayer graphene achieved the best sensitivity of $3058.22 \mathrm{~nm} / \mathrm{RIU}$. Recently, Yu et al. [70] proposed a D-shaped fiber SPR sensor for glucose detection with composite nanomaterial of $\mathrm{MoS}_{2}$-graphene. In this work, the good photoelectric properties of the $\mathrm{MoS}_{2}$-graphene composite nanostructure and the ability of pyrene-1-boronic acid (PBA) to specifically bind glucose molecules were achieved. The experimental result showed that the sensitivity of the SPR sensor could be up to $6708.87 \mathrm{~nm} / \mathrm{RIU}$ when the sensor was modified with three-layer $\mathrm{MoS}_{2}$ and monolayer graphene.

In addition to glucose, protein detection is a basic and important function in biomedicine with outstanding significance. Sharma et al. [71] performed a fiber evanescent wave sensor that took graphene as an absorption-enhancing layer to measure hemoglobin concentration. The result showed the detection limit of $18 \mu \mathrm{g} / \mathrm{dL}$ and sensitivity of $6.71 \times 10^{-4} \mathrm{per} \mathrm{g} / \mathrm{dL}$ at $1000 \mathrm{~nm}$. Different from graphene-based biochemical sensors, the presence of GO could also provide the basis for 
sensor bio-functionalization. For instance, researchers demonstrated a sensitivity enhanced SPR immunosensor based on the GO composite and staphylococcal protein A (SPA) co-modified PCF. Experimental results indicated that the RI sensitivity of the GO modified Au-PCF SPR sensor reached $4649.8 \mathrm{~nm} / \mathrm{RIU}$, which was about $1888 \mathrm{~nm} / \mathrm{RIU}$ higher than that without the GO film, and the human $\mathrm{IgG}$ detection limit reached as low as $10 \mathrm{ng} / \mathrm{ml}$ [72]. Liu et al. [48] developed a human hemoglobin detection sensor using GO nanosheets functionalized LPG, whose intensity was induced by the adsorption of hemoglobin molecules onto GO. This sensor exhibited ultrahigh sensitivity of $1.9 \mathrm{~dB} /(\mathrm{mg} / \mathrm{mL})$ for hemoglobin detection with the limit detection of $0.05 \mathrm{mg} / \mathrm{ml}$. Wang et al. [73] reported a $\mathrm{GO} /$ silver coated polymer cladding fiber biosensor for detecting human IgG in this work, and goat anti-human IgG and SPA were immobilized on the surface of the GO, so that it could be used as the SPR fiber biosensor for detecting the concentration of human IgG. And a high sensitivity of $0.4985 \mathrm{~nm} /(\mu \mathrm{g} / \mathrm{mL})$ and a low limit of detection (LOD) of $0.04 \mu \mathrm{g} / \mathrm{mL}$ were observed. In order to further improve the sensing performance based on the LPG, Esposito et al. [74] combined the LPG with a multilayer system consisting of polycarbonate film and much thinner layer of GO. The multilayer system was conducted for tuning the LPG working point in the highest sensitivity region of mode transition, with a sensitivity of around $2000 \mathrm{~nm} / \mathrm{RIU}$. And They achieved the detection of biotinylated BSA concentrations in range $0.1 \mathrm{aM}-1000 \mathrm{aM}$ with a LOD lower than $0.2 \mathrm{aM}$.

Graphene and its oxides have strong adsorption capacity for compounds with exposed cyclic structures, thus they are prone to bind some molecules with special structures. The bases in DNA contain a six-membered ring structure and graphene have strong $\pi-\pi$ and hydrophobic interactions with exposed bases to adsorbing DNA, which prompts a series of DNA sensors to arise. For example, Qiu et al. [67] reported a evanescent wave absorption sensor based on the micromultimode-fiber coated with the monolayer graphene film, and the experimental result showed a reasonable linear relationship between the value of absorbance and double-stranded DNA concentrations in the range of $5 \mu \mathrm{M}-400 \mu \mathrm{M}$. Later, Yao et al. [35] proposed a novel "FRET on fiber" concept, in which a prGO film was deposited on a fiber-optic modal interferometer, acting as both the fluorescent quencher for the FRET and the sensitive cladding for optical phase measurement due to refractive index changes in biochemical detection, as shown in Fig. 5(b). The result showed a good selectivity for metal ion, dopamine, and ssDNA, with detection limits of $1.2 \mathrm{nM}, 1.3 \mu \mathrm{M}$, and $1 \mathrm{pM}$, respectively. Based on the similar sensing mechanism, they proposed an optical FP cavity to improve the sensing performance with partially reduced $\mathrm{GO}$ (prGO) deposited on the inner wall. With different prGO functional environments, the binding competition between Rh6g and the target molecules (DA, nicotine, and DNA) offers chemical selectivity for the FRET to achieve the selectivity of different biomolecules [5]. In addition, they adopted noise canceled beating and locked-in heterodyne detection with single $\mathrm{Hz}$ precision to measure and amplify the signal of inter-mode crossing, which improved the measurement of single molecular dynamics, as shown in Fig. 5(c).

Furthermore, graphene-fiber sensors could also be used for small molecule detection. For example, Zhou et al. [75] designed an improved $\gamma$-aminobutyric acid (GABA) sensing approach by combining the biorecognition elements with graphene-fiber composite waveguide. In this work, GABA antibody was immobilized on GO as the 
biorecognition element. It demonstrated a sensitivity of $1.03 \mathrm{~nm} / \log \mathrm{M}$ and a lowest LOD of $2.91 \times 10^{-18} \mathrm{M}$, which was 7 orders of magnitude higher than that without the GO interface. Semwal et al. [68] proposed a highly sensitive cholesterol fiber sensor utilizing cholesterol oxidase based on combined phenomenon of localized and propagating surface plasmons. They found that enzyme immobilized over silver, GO, and silver nanoparticles coated unclad fiber had the sensitivity and LOD of $5.14 \mathrm{~nm} / \mathrm{mM}$ and $1.131 \mathrm{mM}$, showing the best performance in three types of SPR based fiber optic cholesterol sensors, as illustrated in Fig. 5(d). Besides, the graphene-fiber sensors show an extensive range of applications, which could also be used to detect ethanol [76], human erythrocyte [77], sucrose [78], dopamine [79], and so on.

(a)
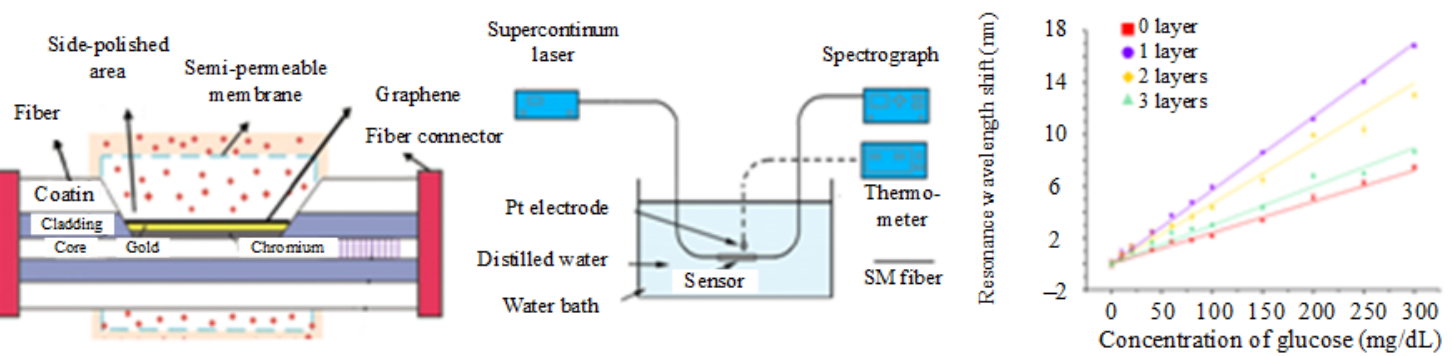

(b)
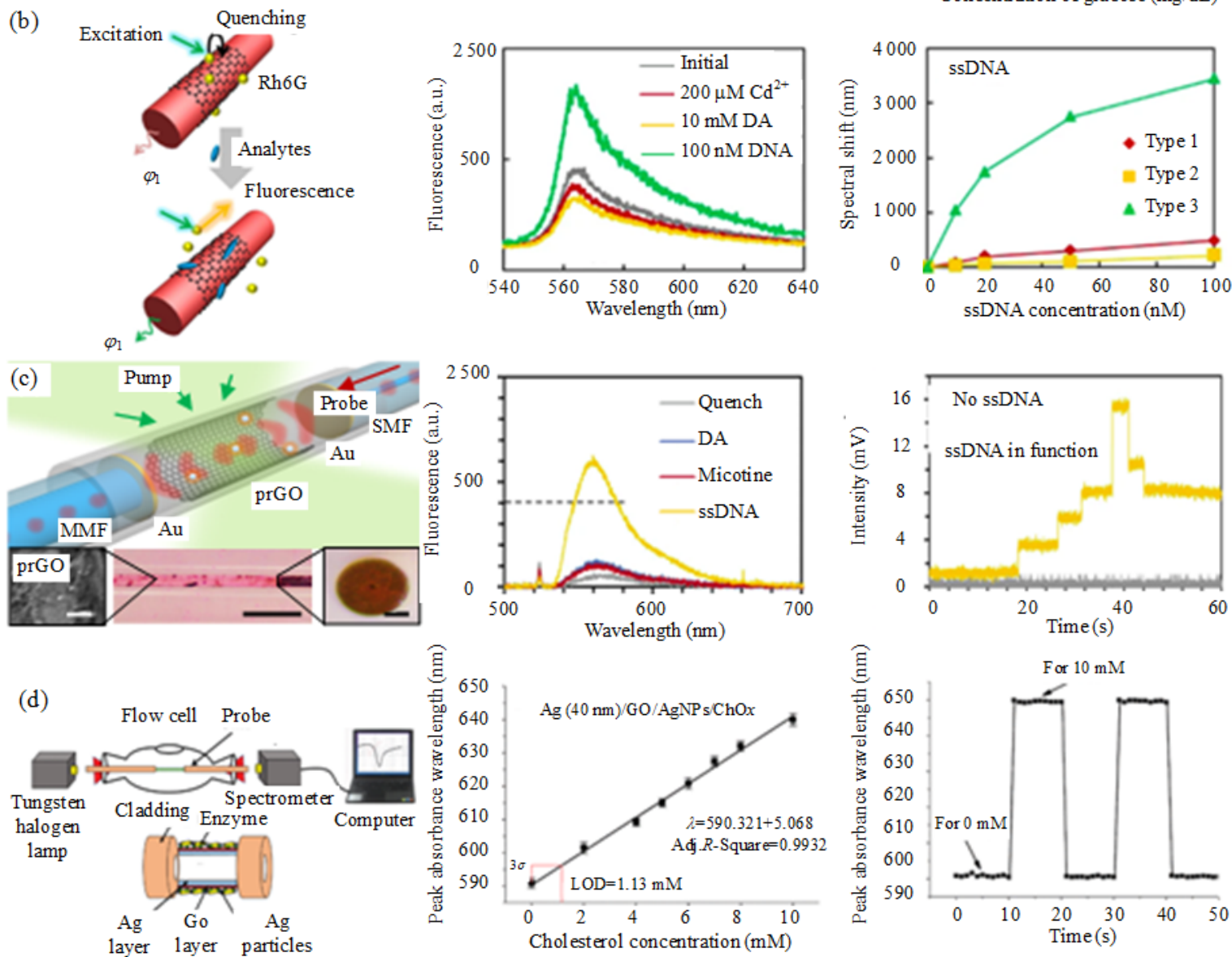

Fig. 5 Graphene-fiber biosensors: (a) gold-graphene/D-shaped fiber sensor for glucose [69], (b) prGO/microfiber sensor with selectivity [35], (c) prGO/microcavity sensor with individual molecule selectivity and sensitivity [5], and (d) silver nanoparticles coated GO/unclad fiber for Cholesterol sensor [68]. 


\section{Conclusions and outlooks}

This article reviews and summarizes the principles, structures, applications, and performances of graphene-based fiber biochemical sensor devices, which have attracted intense interests and exhibited broad application potentials for chemical industry and biomedicine. The comparison of sensing performances for gas sensors and biosensors are shown in Tables 1 and 2 . However, the improvements of sensor sensitivity and selective function are still research hotspots in the future. Therefore, graphene-based fiber sensors are expected to develop in the following areas.

(1) Developing towards single-molecule sensing accuracy in recent years, some new sensing technologies with ultra-high sensitivity have been proposed, such as frequency comb spectroscopy [80, 81] and exceptional points (EPs) [82, 83]. It provides a very good research idea for the realization of single molecular weight graphenefiber composite waveguide sensors. At the same time, the detection means can also be improved, such as the introduction of locked-in heterodyne detection to amplify the small signals generated during the movement of single molecules, improving the sensitivity of the sensor [5].

(2) Development towards multi-parameter sensing measurement, such as biochemical modification and multiple doping.

(3) Sensing in the mid-infrared to the terahertz region [84]. The mid-infrared range is particularly suitable for biosensing because it covers molecular vibrations and can uniquely identify the biochemical components of life, such as proteins, lipids, and DNA. Specifically, biosensing is an area where graphene tunability and infrared light positioning provide huge opportunities.

(4) At present, the sensing theory and technology based on distributed fiber sensors have been very mature [85-87], but its sensitivity is greatly limited by the intrinsic properties. Based on the excellent photoelectric properties of two-dimensional materials, the hybrid structures of distributed fiber sensors and two-dimensional materials will further improve the sensing performance, such as sensing distance and sensitivity.

(5) Combining with the existing AI technology. The detection of small changes in the sensing process can be directly visualized through computer processing without being affected by the accuracy of the detection instrument, which plays a key role in the future development of the detector [88].

Table 1 Summary of sensing performances for gas sensors.

\begin{tabular}{|c|c|c|c|c|c|}
\hline Materials and structure of fiber & Gas type & Detection range & Sensitivity & Recovery/response time & Ref. \\
\hline Graphene/microfiber & Acetone & $580 \mathrm{ppm}-1750 \mathrm{ppm}$ & $0.31 \mathrm{~dB} / \mathrm{ppk}$ & $60 \mathrm{~s} / 80 \mathrm{~s}$ & [53] \\
\hline Graphene/ microfiber & $\mathrm{NH}_{3}$ & $0 \mathrm{ppm}-360 \mathrm{ppm}$ & $\sim 6 \mathrm{pm} / \mathrm{ppm}$ & No Given/0.4 s & [54] \\
\hline Graphene/D-shaped fiber & $\mathrm{NH}_{3} / \mathrm{H}_{2} \mathrm{O}$ & 0 ppm-2000 ppm & $\begin{array}{l}3 \mathrm{pm} / \mathrm{ppm}-\mathrm{NH}_{3} \\
1 \mathrm{pm} / \mathrm{ppm}-\mathrm{H}_{2} \mathrm{O}\end{array}$ & No Given $/<20 \mathrm{~s}$ & [57] \\
\hline Graphene/PCF & $\mathrm{H}_{2} \mathrm{~S}$ & 0 ppm-45 ppm & $0.03143 \mathrm{~nm} / \mathrm{ppm}$ & $80 \mathrm{~s} / 60 \mathrm{~s}$ & [58] \\
\hline $\mathrm{PCF} / \mathrm{rGO}$ & $\mathrm{H}_{2} \mathrm{O}$ & $30 \% \mathrm{RH}-90 \% \mathrm{RH}$ & $0.22 \mathrm{~dB} / \% \mathrm{RH}$ & $\sim 8.1 \mathrm{~s} / \sim 5.2 \mathrm{~s}$ & [89] \\
\hline rGO/D-shaped fiber & Toluene & $40 \mathrm{ppm}-196 \mathrm{ppm}$ & $0.0004622 \mathrm{~dB} / \mathrm{ppm}$ & $256 \mathrm{~s} / 256 \mathrm{~s}$ & [90] \\
\hline $\mathrm{GO} /$ microfiber & Ethanol vapor & $0 \mathrm{ppm}-80 \mathrm{ppm}$ & $\sim 0.138 \mathrm{~nm} / \mathrm{ppm}$ & No Given & [65] \\
\hline $\mathrm{GO} /$ microcavity & $\mathrm{NH}_{3}$ & $1 \mathrm{ppb}-370 \mathrm{ppm}$ & $\sim 200 \mathrm{kHz} / \mathrm{ppm}$ & $18 s-30 s / 15 s-18 s$ & [20] \\
\hline
\end{tabular}


Table 2 Summary of sensing performances for biosensors.

\begin{tabular}{|c|c|c|c|c|c|}
\hline Materials structure of fiber & Liquid & Detection range & Detection limit & Sensitivity & Ref. \\
\hline Graphene based chalcogenide fiber & Hemoglobin & $>18 \mu \mathrm{g} / \mathrm{L}$ & $18 \mu \mathrm{g} / \mathrm{dL}$ & $6.71 \times 10^{-4}$ per $g / d L$ & [71] \\
\hline Graphene-gold/D-shaped fiber & ssDNA & $1 \mathrm{pM}-10 \mu \mathrm{M}$ & $1 \mathrm{pM}$ & $1039.18 \mathrm{~nm} \mathrm{RIU}^{-1}$ & [91] \\
\hline Graphene/D-shaped PCF & Erythrocyte & $0 \mathrm{ppm}$ to $10^{4} \mathrm{ppm}$ & Sub ppm level. & $>1 \mathrm{pm} / \mathrm{ppm}$ & [77] \\
\hline $\mathrm{GO} /$ microfiber & $\gamma$-aminobutyric acid & $10^{-20} \mathrm{~mol} / \mathrm{L}-10^{-5} \mathrm{~mol} / \mathrm{L}$ & $2.91 \times 10^{-18} \mathrm{M}$ & $1.03 \mathrm{~nm} / \log \mathrm{M}$ & [75] \\
\hline $\begin{array}{l}\text { GO and SPA co-modified photonic } \\
\text { crystal fiber }\end{array}$ & $\operatorname{IgG}$ & $0.01 \mu \mathrm{g} / \mathrm{mL}-50 \mu \mathrm{g} / \mathrm{mL}$ & $10 \mathrm{ng} / \mathrm{mL}$ & $4649.8 \mathrm{~nm} \mathrm{RIU}^{-1}$ & [72] \\
\hline \multirow{3}{*}{$\mathrm{prGO} /$ microcavity } & ssDNA & $0 \mathrm{nM}-100 \mathrm{nM}$ & \multirow{3}{*}{ Single molecule } & $8.8 \mathrm{kHz} / \mathrm{nM}$ & \\
\hline & DA & $0 \mathrm{nM}-10 \mathrm{mM}$ & & $0.51 \mathrm{kHz} / \mu \mathrm{M}$ & {$[5]$} \\
\hline & Nicotine & $0-1.24 \mu \mathrm{M}$ & & $0.2 \mathrm{kHz} / \mathrm{nM}$ & \multirow{4}{*}[35]{} \\
\hline \multirow{3}{*}{$\mathrm{prGO} /$ microfiber } & Metal ion & $0 \mu \mathrm{M}-10 \mu \mathrm{M}$ & $1.1 \mathrm{nM}$ & $90 \mathrm{pm} / \mu \mathrm{M}$ & \\
\hline & Dopamine & $0 \mathrm{mM}-10 \mathrm{mM}$ & $1.3 \mu \mathrm{M}$ & No Given & \\
\hline & ssDNA & $0 \mathrm{nM}-10 \mathrm{nM}$ & $1 \mathrm{pM}$ & $\sim 100 \mathrm{pm} / \mathrm{nM}$ & \\
\hline
\end{tabular}

Open Access This article is distributed under the terms of the Creative Commons Attribution 4.0 International License (http://creativecommons.org/licenses/by/4.0/), which permits unrestricted use, distribution, and reproduction in any medium, provided you give appropriate credit to the original author(s) and the source, provide a link to the Creative Commons license, and indicate if changes were made.

\section{References}

[1] B. Yao, Y. Wu, Z. Wang, Y. Cheng, Y. Rao, Y. Gong, et al., "Demonstration of complex refractive index of graphene waveguide by microfiber-based Mach-Zehnder interferometer," Optics Express, 2013, 21(24): 29818-29826.

[2] V. Semwal and B. D. Gupta, "Highly sensitive surface plasmon resonance based fiber optic $\mathrm{pH}$ sensor utilizing rGO-Pani nanocomposite prepared by in situ method," Sensors and Actuators B: Chemical, 2019, 283: 632-642.

[3] H. Ting and S. C. Kin, "Graphene-based ammonia-gas sensor using in-fiber Mach-Zehnder interferometer," IEEE Photonics Technology Letters, 2017, 29(23): 2035-2038.

[4] W. Xu, T. Yang, F. Qin, D. Gong, Y. Du, and G. Dai, "A sprayed graphene pattern-based flexible strain sensor with high sensitivity and fast response," Sensors (Switzerland), 2019, 19(5): 1-11.

[5] Z. Cao, B. Yao, C. Qin, R. Yang, Y. Guo, Y. Zhang, et al., "Biochemical sensing in graphene-enhanced microfiber resonators with individual molecule sensitivity and selectivity," Light: Science \& Applications, 2019, 8(1): 4-13.

[6] J. A. Kim, T. Hwang, S. R. Dugasani, R. Amin, A. Kulkarni, S. H. Park, et al., "Graphene based fiber optic surface plasmon resonance for bio-chemical sensor applications," Sensors and Actuators B: Chemical, 2013, 187: 426-433.

[7] B. N. Shivananju, W. Yu, Y. Liu, Y. Zhang, B. Lin, et al., "The roadmap of graphene-based optical biochemical sensors," Advanced Functional Materials., 2017, 27(19): 1-19.

[8] H. Chen, R. Li, and F. Xu, "Optical microfiber sensors: sensing mechanisms, and recent advances," Journal of Lightwave Technology, 2019, 37(11): 2577-2589.

[9] L. Tong, "Micro/nanofibre optical sensors: challenges and prospects," Sensors (Switzerland), 2018, 18(3): 903.

[10] W. Choi, I. Lahiri, R. Seelaboyina, and Y. S. Kang, "Synthesis of graphene and its applications: a review," Critical Reviews in Solid State and Materials Sciences, 2010, 35(1): 52-71.

[11] A. K. Geim, "Graphene: status and prospects," Science, 2009, 324(5934): 1530-1534.

[12] M. J. Allen, V. C. Tung, and R. B. Kane, "Honeycomb carbon: a review of graphene," Chemical Reviews, 2010, 110(1): 132-145.

[13] F. Bonaccorso, Z. Sun, T. Hasan, and A. C. Ferrari, "Graphene photonics and optoelectronics," Nature Photonics, 2010, 4(9): 611-622.

[14] R. J. Young, I. A. Kinloch, L. Gong, and K. S. Novoselov, "The mechanics of graphene nanocomposites: a review," Composites Science and Technology, 2012, 72(12): 1459-1476.

[15] H. Chang and H. Wu, "Graphene-based nanomaterials: synthesis, properties, and optical and optoelectronic applications," Advanced Functional Materials, 2013, 23(16): 1984-1997.

[16] W. Li, B. Chen, C. Meng, W. Fang, Y. Xiao, X. Li, et al., "Ultrafast all-optical graphene modulator," Nano Letters, 2014, 14(2): 955-959. 
[17] A. Martinez and Z. Sun, "Nanotube and graphene saturable absorbers for fibre lasers," Nature Photonics, 2013, 7(11): 842-845.

[18] B. Yao, Y. Liu, S. W. Huang, C. Choi, Z. Xie, J. F. Flores, et al., "Broadband gate-tunable terahertz plasmons in graphene heterostructures," Nature Photonics, 2018, 12(1): 22-28.

[19] S. Y. Hong, J. I. Dadap, N. Petrone, P. C. Yeh, J. Hone, and R. M. Osgood, "Optical third-harmonic generation in graphene," Physical Review X, 2013, 3(2): 021014.

[20] B. Yao, C. Yu, Y. Wu, S. W. Huang, H. Wu, Y. Gong, et al., "Graphene-enhanced Brillouin optomechanical microresonator for ultrasensitive gas detection," Nano Letters, 2017, 17(8): 4996-5002.

[21]N. An, T. Tan, Z. Peng, C. Qin, Z. Yuan, L. Bi, et al., "Electrically tunable four-wave-mixing in graphene heterogeneous fiber for individual gas molecule detection," Nano Letters, 2020, 20(9): 6473-6480.

[22] A. H. Castro Neto, F. Guinea, N. M. R. Peres, K. S. Novoselov, and A. K. Geim, "The electronic properties of graphene," Reviews of Modern Physics, 2009, 81(1): 109-162.

[23] S. A. Mikhailov and K. Ziegler, "New electromagnetic mode in graphene," Physical Review letters, 2007, 99(1): 1-4.

[24] P. Zheng and N. Wu, "Fluorescence and sensing applications of graphene oxide and graphene quantum dots: a review," Chemistry - An Asian Journal, 2017, 12(18): 2343-2353.

[25] P. Avouris, Z. Chen, and V. Perebeinos, "Carbon-based electronics," Nature Nanotechnology, 2007, 2(10): 605-615.

[26] Y. Wu, B. Yao, C. Yu, and Y. Rao, "Optical graphene gas sensors based on microfibers: a review," Sensors (Switzerland), 2018, 18(4): 941.

[27] A. Das, S. Pisana, B. Chakraborty, S. Piscanec, S. K. Saha, U. V. Waghmare, et al., "Monitoring dopants by Raman scattering in an electrochemically top-gated graphene transistor," Nature Nanotechnology, 2008, 3(4): 210-215.

[28] U. Sampath, D. Kim, and M. Song, "Hemoglobin detection using a graphene oxide functionalized side-polished fiber sensor," in SPIE Optics + Optoelectronics, Prague, Apirl, 2019, pp. 82.

[29] S. E. U. Lima, R. G. Farias, F. M. Araújo, L. A. Ferreira, J. L. Santos, V. Miranda, et al., "Fiber laser sensor based on a phase-shifted chirped grating for acoustic sensing of partial discharges," Photonic Sensors, 2013, 3(1): 44-51.

[30] A. N. Grigorenko, M. Polini, and K. S. Novoselov, "Graphene plasmonics," Nature Photonics, 2012, 6(11): 749-758.

[31] W. Wei, J. Nong, Y. Zhu, G. Zhang, N. Wang, S. Luo, et al., "Graphene/Au-enhanced plastic clad silica fiber optic surface plasmon resonance sensor," Plasmonics, 2018, 13(2): 483-491.
[32] X. Yang, Z. Sun, T. Low, H. Hu, X. Guo, F. J. G. de Abajo, et al., "Nanomaterial-based plasmonenhanced infrared spectroscopy," Advanced Materials, 2018, 30(20): 1704896.

[33] G. X. Ni, A. S. McLeod, Z. Sun, L. Wang, L. Xiong, K. W. Post, et al., "Fundamental limits to graphene plasmonics," Nature, 2018, 557(7706): 530-533.

[34] R. B. Sekar and A. Periasamy, "Fluorescence resonance energy transfer (FRET) microscopy imaging of live cell protein localizations," The Journal of Cell Biology, 2003, 160(5): 629-633.

[35]B. Yao, Y. Wu, C. Yu, J. He, Y. Rao, Y. Gong, et al., "Partially reduced graphene oxide based FRET on fiber-optic interferometer for biochemical detection," Scientific Reports, 2016, 6: 23706.

[36] P. Suvarnaphaet and S. Pechprasarn, "Graphenebased materials for biosensors: a review," Sensors (Switzerland), 2017, 17(10): 2161.

[37] X. Li, W. Cai, J. An, S. Kim, J. Nah, D. Yang, et al., "Large-area synthesis of high-quality and uniform graphene films on copper foils," Science, 2009, 324(5932): 1312-1314.

[38]D. Parviz, F. Irin, S. A. Shah, S. Das, C. B. Sweeney, and M. J. Green, "Challenges in liquid-phase exfoliation, processing, and assembly of pristine graphene," Advanced Materials, 2016, 28(40): 8796-8818.

[39] M. Yi and Z. Shen, "A review on mechanical exfoliation for the scalable production of graphene," Journal of Materials Chemistry A, 2015, 3(22): 11700-11715.

[40] V. Sharma, Y. Jain, M. Kumari, R. Gupta, S. K. Sharma, S. K. Sharma, et al., "Synthesis and characterization of graphene oxide (GO) and reduced graphene oxide (rGO) for gas sensing application," Macromolecular Symposia, 2017, 376(1): 1-5.

[41] R. Muñoz and C. Gómez-Aleixandre, "Review of CVD synthesis of graphene," Chemical Vapor Deposition, 2013, 19(10-11-12): 297-322.

[42] S. Perumbilavil, P. Sankar, T. Priya Rose, and R. Philip, "White light Z-scan measurements of ultrafast optical nonlinearity in reduced graphene oxide nanosheets in the 400-700 nm region," Applied Physics Letters, 2015, 107(5): 051104.

[43] T. Tan, X. Jiang, C. Wang, B. Yao, and H. Zhang, "2D material optoelectronics for information functional device applications: status and challenges," Advanced Science, 2020, 7(11): 2000058.

[44] K. Chen, X. Zhou, X. Cheng, R. Qiao, Y. Cheng, C. Liu, et al., "Graphene photonic crystal fibre with strong and tunable light-matter interaction," Nature Photonics, 2019, 13(11): 754-759.

[45] J. Ma, W. Jin, H. L. Ho, and J. Y. Dai, "High-sensitivity fiber-tip pressure sensor with graphene diaphragm," Optics Letters, 2012, 37(13): 
2493-2495.

[46] S. Y. Choi, D. K. Cho, Y.-W. Song, K. Oh, K. Kim, et al., "Graphene-filled hollow optical fiber saturable absorber for efficient soliton fiber laser mode-locking," Optics Express, 2012, 20(5): 5652-5657.

[47] J. Kou, J. Chen, Y. Chen, F. Xu, and Y. Lu, "Platform for enhanced light-graphene interaction length and miniaturizing fiber stereo devices," Optica, 2014, 1(5): 207-310.

[48] C. Liu, B. Xu, L. Zhou, Z. Sun, H. Mao, J. Zhao, et al., "Graphene oxide functionalized long period fiber grating for highly sensitive hemoglobin detection," Sensors and Actuators B: Chemical, 2018, 261: 91-96.

[49] M. Gorji, A. Sadeghianmaryan, H. Rajabinejad, S. Nasherolahkam, and X. Chen, "Development of highly $\mathrm{pH}$-sensitive hybrid membranes by simultaneous electrospinning of amphiphilic nanofibers reinforced with graphene oxide," Journal of Functional Biomaterials, 2019, 10(2): 23.

[50] M. B. Hossain, M. M. Islam, L. F. Abdulrazak, M. M. Rana, T. B. A. Akib, and M. Hassan, "Graphene-coated optical fiber SPR biosensor for BRCA1 and BRCA2 breast cancer biomarker detection: a numerical design-based analysis," Photonic Sensors, 2020, 10(1): 67-79.

[51]A. Syuhada, M. S. Shamsudin, S. Daud, G. Krishnan, S. W. Harun, and M. S. Abd Aziz, "Single-mode modified tapered fiber structure functionalized with GO-PVA composite layer for relative humidity sensing," Photonic Sensors, DOI: 10.1007/s13320-020-0595-0.

[52] A. Zhang, Y. Wu, B. Yao, and Y. Gong, "Optimization study on graphene-coated microfiber Bragg grating structures for ammonia gas sensing," Photonic Sensors, 2015, 5(1): 84-90.

[53] B. Yao, Y. Wu, Y. Chen, X. Liu, Y. Gong, and Y. Rao, "Graphene-based microfiber gas sensor," in OFS2012 22nd International Conference on Optical Fiber Sensor, Beijing, 2012, pp. 8421CD-1-8421CD-4.

[54] B. Yao, Y. Wu, Y. Cheng, A. Zhang, Y. Gong, Y. J. Rao, et al., "All-optical Mach-Zehnder interferometric $\mathrm{NH}_{3}$ gas sensor based on graphene/microfiber hybrid waveguide," Sensors and Actuators B: Chemical, 2014, 194: 142-148.

[55] B. Yao, Y. Wu, A. Zhang, F. Wang, Y. Rao, Y. Gong, et al., "Graphene Bragg gratings on microfiber," Optics Express, 2014, 22(20): 23829-23835.

[56] B. Yao, Y. Wu, A. Zhang, Y. Rao, Z. Wang, Y. Cheng, et al., "Graphene enhanced evanescent field in microfiber multimode interferometer for highly sensitive gas sensing," Optics Express, 2014, 22(23): 28154-28162.

[57] Y. Wu, B. Yao, A. Zhang, X. Cao, Z. Wang, Y. Rao, et al., "Graphene-based D-shaped fiber multicore mode interferometer for chemical gas sensing," Optics Letters, 2014, 39(20): 6030-6033.

[58] X. Feng, W. Feng, C. Tao, D. Deng, X. Qin, and R. Chen, "Hydrogen sulfide gas sensor based on graphene-coated tapered photonic crystal fiber interferometer," Sensors and Actuators B: Chemical, 2017, 247: 540-545.

[59] D. Pawar, B. V. B. Rao, and S. N. Kale, " $\mathrm{Fe}_{3} \mathrm{O}_{4}$-decorated graphene assembled porous carbon nanocomposite for ammonia sensing: study using an optical fiber Fabry-Perot interferometer," Analyst, 2018, 143(8): 1890-1898.

[60] S. Sridevi, K. S. Vasu, N. Bhat, S. Asokan, and A. K. Sood, "Ultra sensitive $\mathrm{NO}_{2}$ gas detection using the reduced graphene oxide coated etched fiber Bragg gratings," Sensors and Actuators B: Chemical, 2016, 223: 481-486.

[61] Y. Zhang, Y. Chen, K. Zhou, C. Liu, J. Zeng, H. Zhang, et al., "Improving gas sensing properties of graphene by introducing dopants and defects: a first-principles study," Nanotechnology, 2009, 20(18): 185504.

[62] C. Yu, Y. Wu, X. Liu, F. Fu, Y. Gong, Y. J. Rao, et al., "Miniature fiber-optic $\mathrm{NH}_{3}$ gas sensor based on Pt nanoparticle-incorporated graphene oxide," Sensors and Actuators B: Chemical, 2017, 244: 107-113.

[63] Y. Wang, C. Shen, W. Lou, and F. Shentu, "Fiber optic humidity sensor based on the graphene oxide/PVA composite film," Optics Communications, 2016, 372: 229-234.

[64]H. Fu, Y. Jiang, J. Ding, J. Zhang, M. Zhang, Y. Zhu, et al., "Zinc oxide nanoparticle incorporated graphene oxide as sensing coating for interferometric optical microfiber for ammonia gas detection," Sensors and Actuators B: Chemical, 2018, 254: 239-247.

[65] J. Zhang, H. Fu, J. Ding, M. Zhang, and Y. Zhu, "Graphene-oxide-coated interferometric optical microfiber ethanol vapor sensor," Applied Optics, 2017, 56(31): 8828-8831.

[66] S. Sridevi, K. S. Vasu, S. Asokan, and A. K. Sood, "Sensitive detection of C-reactive protein using optical fiber Bragg gratings," Biosensors and Bioelectronics, 2015, 65: 251-256.

[67] H. Qiu, S. Gao, P. Chen, Z. Li, X. Liu, C. Zhang, et al., "Evanescent wave absorption sensor based on tapered multimode fiber coated with monolayer graphene film," Optics Communications, 2016, 366: 275-281.

[68] V. Semwal and B. D. Gupta, "LSPR- and SPR-based fiber-optic cholesterol sensor using immobilization of cholesterol oxidase over silver nanoparticles coated graphene oxide nanosheets," IEEE Sensors Journal, 2017, 18(3): 1039-1046.

[69] P. Zhang, B. Lu, Y. Sun, H. Yu, K. Xu, and D. Li, 
"Side-polished flexible SPR sensor modified by graphene with in situ temperature self-compensation," Biomedical Optics Express, 2019, 10(1): 215-225.

[70] H. Yu, Y. Chong, P. Zhang, J. Ma, and D. Li, “A D-shaped fiber SPR sensor with a composite nanostructure of $\mathrm{MoS}_{2}$-graphene for glucose detection," Talanta, 2020, 219: 121324.

[71] A. K. Sharma and J. Gupta, "Graphene based chalcogenide fiber-optic evanescent wave sensor for detection of hemoglobin in human blood," Optical Fiber Technology, 2018, 41: 125-130.

[72] Q. Wang and B. Wang, "Sensitivity enhanced SPR immunosensor based on graphene oxide and SPA co-modified photonic crystal fiber," Optics \& Laser Technology, 2018, 107: 210-215.

[73] Q. Wang and B. T. Wang, "Surface plasmon resonance biosensor based on graphene oxide/silver coated polymer cladding silica fiber," Sensors and Actuators B: Chemical, 2018, 275: 332-338.

[74] F. Esposito, L. Sansone, C. Taddei, S. Campopiano, M. Giordano, and A. Iadicicco, "Ultrasensitive biosensor based on long period grating coated with polycarbonate-graphene oxide multilayer," Sensors and Actuators B: Chemical, 2018, 274: 517-526.

[75] J. Zhou, Y. Huang, C. Chen, A. Xiao, T. Guo, and B. O. Guan, "Improved detection sensitivity of $\gamma$-aminobutyric acid based on graphene oxide interface on an optical microfiber," Physical Chemistry Chemical Physics, 2018, 20(20): 14117-14123.

[76] A. Aziz, H. N. Lim, S. H. Girei, M. H. Yaacob, M. A. Mahdi, N. M. Huang, et al., "Silver/graphene nanocomposite-modified optical fiber sensor platform for ethanol detection in water medium," Sensors and Actuators B: Chemical, 2015, 206: 119-125.

[77] B. Yao, Y. Wu, D. J. Webb, J. Zhou, Y. Rao, A. Pospori, et al., "Graphene-based D-shaped polymer FBG for highly sensitive erythrocyte detection," IEEE Photonics Technology Letters, 2015, 27(22): 2399-2402.

[78] J. K. Nayak, P. Parhi, and R. Jha, "Graphene oxide encapsulated gold nanoparticle based stable fibre optic sucrose sensor," Sensors and Actuators B: Chemical, 2015, 221: 835-841.

[79] W. Hu, Y. Huang, C. Chen, Y. Liu, T. Guo, and B. O. Guan, "Highly sensitive detection of dopamine using a graphene functionalized plasmonic fiber-optic sensor with aptamer conformational amplification," Sensors and Actuators B: Chemical, 2018, 264: 440-447.

[80] B. Yao, S. W. Huang, Y. Liu, A. K. Vinod, C. Choi,
M. Hoff, et al., "Gate-tunable frequency combs in graphene-nitride microresonators," Nature, 2018, 558(7710): 410-414.

[81] H. Chen, Q. Ji, H. Wang, Q. Yang, Q. Cao, Q. Gong, et al., "Chaos-assisted two-octave-spanning microcombs," Nature Communications, 2020, 11(1): $1-6$.

[82] J. Zhang, B. Peng, Ş. K. Özdemir, K. Pichler, D. O. Krimer, G. Zhao, et al., "A phonon laser operating at an exceptional point," Nature Photonics, 2018, 12(8): 479-484.

[83] W. Chen, Ş. K. Özdemir, G. Zhao, J. Wiersig, and L. Yang, "Exceptional points enhance sensing in an optical microcavity," Nature, 2017, 548(7666): 192-196.

[84] D. Rodrigo, O. Limaj, D. Janner, D. Etezadi, F. J. García De Abajo, V. Pruneri, et al., "Mid-infrared plasmonic biosensing with graphene," Science, 2015, 349(6244): 165-168.

[85] H. Wu, Z. Wang, F. Peng, Z. Peng, X. Li, Y. Wu, et al., "Field test of a fully distributed fiber optic intrusion detection system for long-distance security monitoring of national borderline," in OFS2014 23rd International Conference on Optical Fiber Sensors, Spain, June 2, 2014, pp. 915790.

[86] Z. Wang, J. Zeng, J. Li, F. Peng, L. Zhang, Y. Zhou, et al., " $175 \mathrm{~km}$ phase-sensitive OTDR with hybrid distributed amplification," in OFS2014 23rd International Conference on Optical Fiber Sensors, Spain, June 2, 2014, pp. 9157D5.

[87] H. Wu, Y. Qian, W. Zhang, and C. Tang, "Feature extraction and identification in distributed optical-fiber vibration sensing system for oil pipeline safety monitoring," Photonic Sensors, 2017, 7(4): 305-310.

[88] T. Tan, C. Peng, Z. Yuan, X. Xie, H. Liu, Z. Xie, et al., "Predicting Kerr soliton combs in microresonators via deep neural networks," Journal of Lightwave Technology, 2020, 38(23): 6591-6599.

[89] R. Gao, D. F. Lu, J. Cheng, Y. Jiang, L. Jiang, and Z. Qi, "Humidity sensor based on power leakage at resonance wavelengths of a hollow core fiber coated with reduced graphene oxide," Sensors and Actuators B: Chemical, 2016, 222: 618-624.

[90] Y. Xiao, J. Yu, L. Shun, S. Tan, X. Cai, Y. Luo, et al., "Reduced graphene oxide for fiber-optic toluene gas sensing," Optics Express, 2016, 24(25): 28290-28302.

[91] N. M. Y. Zhang, K. Li, P. P. Shum, X. Yu, S. Zeng, $\mathrm{Z}$. Wu, et al., "Hybrid graphene/gold plasmonic fiber-optic biosensor," Advanced Materials Technologies, 2017, 2(2): 1600185. 